\title{
Investigation on A Rapid Modelling Analysis Approach of 3D Pentamode Metamaterials
}

\author{
Qian-jin Xiao a, ${ }^{,}$, Ai-guo Zhaob, Zhuo You ${ }^{\mathrm{c}}$ and Lei WANG ${ }^{\mathrm{b}}$ \\ Wuhan Second Ship Design and Research Institute, Wuhan 430205, China \\ axiaoqj1986@qq.com, bshiang37@gmail.com, czhuoyouzju@zju.edu.cn, dywangl@mail.ustc.edu.cn
}

Keywords: Abaqus/Python, Pentamode metamaterial, Rapid modelling analysis.

Abstract. The realization of the special physical properties of metamaterials depends on the artificially designed constitutive relations, and their mechanical properties become the key research point. In order to enhance the analysis efficiency of mechanical properties of three-dimensional (3D) pentamode metamaterials, we propose a rapid modeling method based on Abaqus using the Python script language. The rapid modelling parameters are determined according to the microstructure characteristics of 3D pentamode metamaterials. The parametrized model of pentamode metamaterials has been built by using the Python language and interactive input interfaces. Based on the rapid pre-processing and post-processing modules, the finite element analysis (FEA) model is automatically generated and the analysis and calculation of simulation results are automatically completed. The rapid modelling approach proposed in this paper can conveniently build the FEA model by directly inputting the design parameters of 3D pentamode metamaterials and after calculation, automatically extract needed data and information, make an analysis and calculate through the rapid post-processing module. The research result lays a crucial foundation for further large-scale simulation and optimized design of mechanical properties of 3D pentamode metamaterials.

\section{Introduction}

The metamaterial technology is a new approach emerging in recent years, which adopts the special periodic microstructure design to achieve the artificially designed physical properties ${ }^{[1-4]}$. It has broad application prospect in the fields of optical cloaking, acoustic cloaking and electromagnetics et al. and has already become one of the most popular research topics both at home and abroad ${ }^{[5-9]}$. The metamaterials depend on their special artificially designed constitutive relation to realize the special physical properties, and their mechanical properties attract extensive research attention ${ }^{[10]}$. Abaqus is a software suite offering abundant material constitutive models and unit types and having the powerful secondary development function, thus an effective tool for the mechanical properties analysis of metamaterials. However, the 3D pentamode metamaterials have a complicated space structure $^{[11]}$, so it is low efficient and difficult to realize the $3 \mathrm{D}$ geometric modelling by utilizing the pre-processing module of the Abaqus software. Parameterized modelling by the UG software needs a complex process of import and export of intermediate files, as well as the pre-process of repeated inputs of various parameters and setting multiple dialogue boxes after importing the 3D geometric model. The entire process is repeating and time consuming and greatly affects the efficiency. Therefore, a solution must be proposed for achieving automatic processing functions on Abaqus, such as automatic parametrized modelling, specifying material properties, creating boundary conditions and output, grid partition, calculation and post-processing of analysis results.

Python script language of Abaqus software is an object-oriented programming language ${ }^{[12]}$ that can offer a solution to improve the efficiency of mechanical properties analysis of 3D pentamode metamaterials. If it is used for the modelling analysis, the Abaqus/CAE user interfaces may be bypassed and the specific interactive interfaces can be established as needed, without the trouble of repeated selecting of dialog boxes and child dialog boxes to set parameters, thus the modelling analysis of metamaterials becomes more convenient and efficient. 
The rapid FEA modelling analysis approach for 3D pentamode metamaterials proposed in this paper is based on the Abaqus/Python script language. It builds the FEA model (including geometric modelling, specifying material properties, creating analysis steps and outputs, creating load and boundary conditions, and grid partition etc.) on the interactive parametrized statement and automatic update, to solve a series of problems in the FEA modelling of 3D space entity model, such as description of geometric model, parameter linkage, automatic model updating and automatic post-processing of simulation results. These can greatly improve the modelling analysis efficiency of complicated 3D entity model, and have very practical significance for realizing the automatic processing of large-scale calculations.

\section{Parametric Analysis of Metamaterial Microstructure Model}

Because the microstructure model of 3D pentamode metamaterials is formed by the periodic array of many single cells in three directions: horizontal, longitudinal and vertical, the parameterized design must be created for single cells when building the metamaterial microstructure model, which is obtained through array approach.

\section{Model Parameterization}

As shown in Fig.1, the single cells ${ }^{[11]}$ of $3 \mathrm{D}$ pentamode metamaterials have the cubic lattice structure connected by a number of spindles regularly. Their structural parameters mainly include: lattice constant $a$, node position ratio $p$, cone apex diameter $d$, cone base diameter $D$ and spindle length $h$.

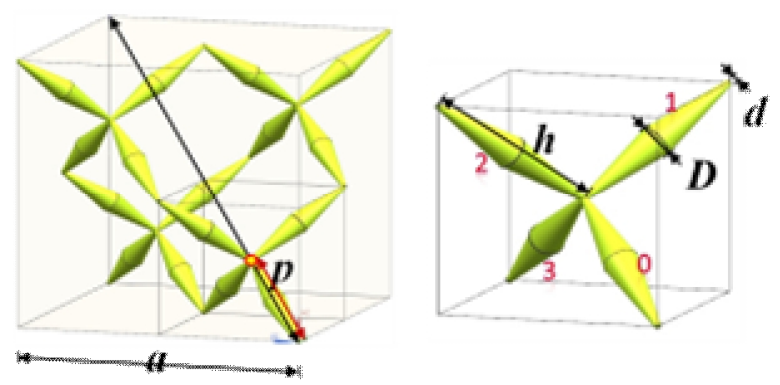
(a) Single cell model
(b) Partial enlargement of single cell

Fig.1 Structural parameters of single cell parametric modelling of 3D pentamode materials

\section{Parametric Analysis}

Lattice constant $\boldsymbol{a}$. It determines the size of pentamode metamaterial single cells.

Node position ratio $p$. It is the ratio of spindle length at the main diagonal line and its length, and determines the symmetry of pentamode metamaterial microstructure. Generally $0<p<0.5$; if and only if $p=0.25$, the microstructure is a symmetrical structure and now the pentamode metamaterials is represented as orthogonal isotropic materials.

Cone apex diameter $\boldsymbol{d}$. It determines the size of spindle end, namely the size of connecting point of pentamode metamaterial microstructure.

Cone base diameter $D$. It determines the size of spindle middle, ranging within $d \leq D<a / 2$.

Spindle length $\boldsymbol{h}$. Since the position of node P moves along the main diagonal line of single cell as the parameter $p$ changes. If the spindles are numbered as shown in Fig.1(b), it is easily concluded that the lengths of 1\#, 2\# and 3\# spindles are equal (but not equal to 0\# spindle length). Obviously, the spindle length $h$ is closely related to $a$ and $p$. 
According to the above analysis, the parameters $a, p, d$ and $D$ are independent parameters and the parameter $h$ is a relevant parameter. As a result, the parametrized model of single cells of 3D pentamode metamaterials only contains four geometric parameters.

\section{Rapid Pre-processing Modelling of Metamaterial}

During the programming process, firstly the "from...import..." statement are used to import modules to call relevant functions or objects; the interactive input interface is set through the "getInputs()" function; the "while" loop statement is used to achieve repetitive operation. For the pre-processing, the geometric modelling and material property definition employ the interactive input interfaces; the pre-processes, such as grid partition, analysis step creation and load and boundary condition setting, are all completed by automatic programming.

\section{Interactive Parametrized Modelling}

To be more convenient and direct for building the single cell model of 3D pentamode metamaterials, a user-defined interactive interface is applied for assigning values to model parameters, programmed as follows by the Python language:

myParameters $=(($ 'Parameter $p(0<p<0.5)=$ ', ' '), ('Parameter $a(m m)=$ ', ' '),

('Parameter $D(m m)=$ ', ' '), ('Parameter $d(m m)=$ ', ' '))

$p, a, D, d=$ getInputs ( fields $=$ myParameters, label $=$ 'Evaluate parameters: $\left.{ }^{\prime}\right)$

$p, a, D, d=$ float $(p)$, float $(a)$, float $(D)$, float $(d)$

Run the above program, the interactive input interface may be obtained as shown in Fig.2.

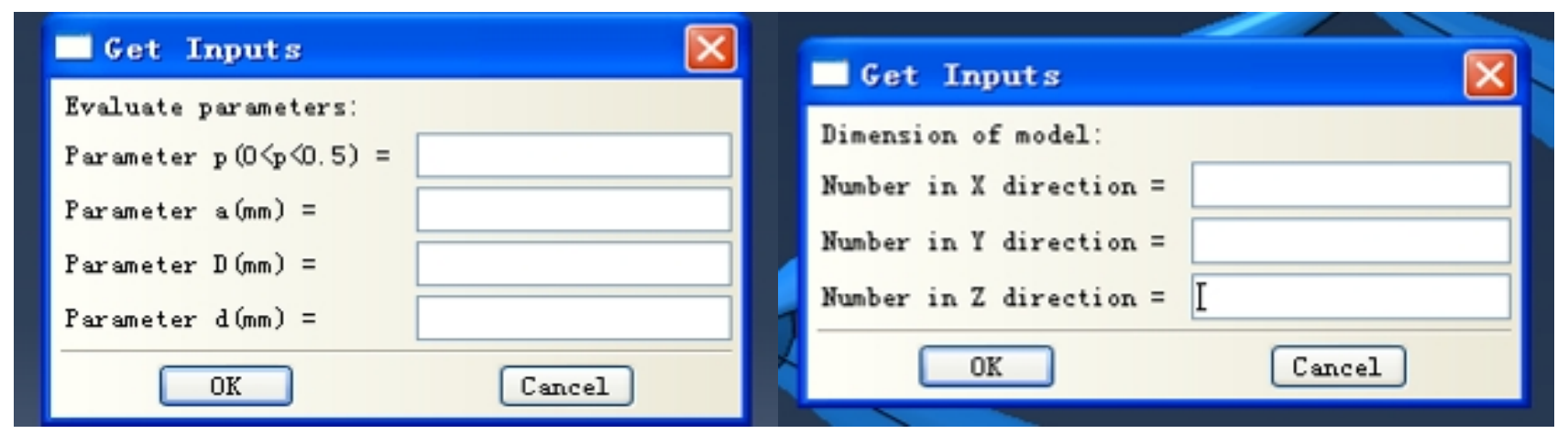

Fig.2 Interactive input of single cell model parameters of metamaterial

Fig.3 Interactive input of metamaterial model dimension

After getting the metamaterial single cell model, the linear array approach is utilized for periodic extension in the three directions: $\mathrm{X}, \mathrm{Y}$ and $\mathrm{Z}$, and finally the metamaterial model is obtained. The number of single cells of metamaterials in each direction is input by the interactive interface, programmed as follows by the Python language:

myDimension $=(($ Number in $X$ direction = ', ' '), ('Number in Y direction = ', ' '),

('Number in $Z$ direction = ', ' '))

$N x x, N y y, N z z=$ getInputs $\left(\right.$ fields $=$ myDimension, label $=$ 'Dimension of model: $\left.:^{\prime}\right)$

$N x, N y, N z=\operatorname{int}(N x x), \operatorname{int}(N y y), \operatorname{int}(N z z)$

Run the above program, the interactive input interface may be obtained as shown in Fig.3.

\section{Result of Parametric Modelling}

Based on the four independent parameters: $a, p, d$ and $D$, the interactive input interfaces are used to directly assign the model parameters, and the structural shapes of pentamode metamaterial single cells under different structural parameters are shown in Fig.4. 


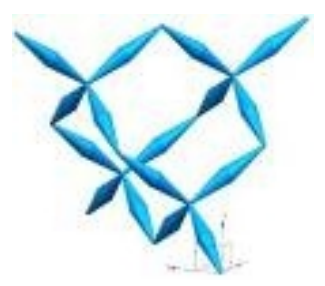

(a) $p=0.25, a=37.3 \mathrm{~mm}$, $D=3 \mathrm{~mm}, d=0.55 \mathrm{~mm}$

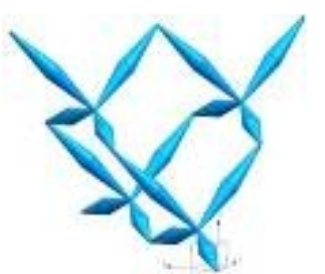

(b) $p=0.15, a=37.3 \mathrm{~mm}$, $D=3 \mathrm{~mm}, d=0.55 \mathrm{~mm}$

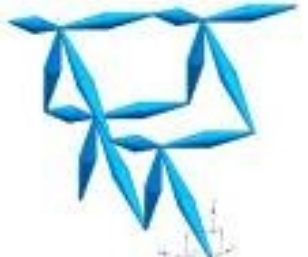

(c) $p=0.41, a=37.3 \mathrm{~mm}$, $D=3 \mathrm{~mm}, d=0.55 \mathrm{~mm}$

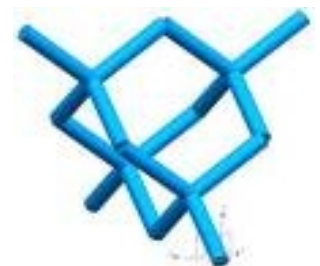

(d) $p=0.25, a=37.3 \mathrm{~mm}$, $D=3 \mathrm{~mm}, d=3 \mathrm{~mm}$

Fig.4 Structural shapes of pentamode metamaterial single cells under different structural parameters

When $p=0.25, a=15 \mathrm{~mm}, D=1.32 \mathrm{~mm}$ and $d=0.71 \mathrm{~mm}$, the parameterized modelling result of a $2 \times 2 \times 4$ pentamode metamaterial model is shown in Fig. 5. It is worth noting that two flat plates at the top and bottom of the metamaterial model are automatically generated by the program.

\section{Interactively Defined Material Properties}

For the sake of defining the base material properties of metamaterials during simulation analysis of mechanical properties, the material properties are defined by the interactive input interface either, programmed as follows by the Python language:

myMaterial $=$ myModel . Material $($ name $=$ material_name $)$

material_property $=\left(\left({ }^{\prime} E(M P a)=\right.\right.$ ',' '), $\left(' v(0<v<0.5)=\right.$,', '),('Density $\left(t / \mathrm{mm}^{\wedge} 3\right)=$, ', ' $\left.\left.^{\prime}\right)\right)$

$E, v$, den $=$ getInputs(fields $=$ material_property, label $=$ 'Define material property: $\left.{ }^{\prime}\right)$

$E, v$, den $=$ float $(E)$, float $(v)$, float (den)

myMaterial. Elastic(table $=((E, v))$,

myMaterial. Density (table $=(($ den, $))$,

Run the above program, the interactive input interface may be obtained as follows:

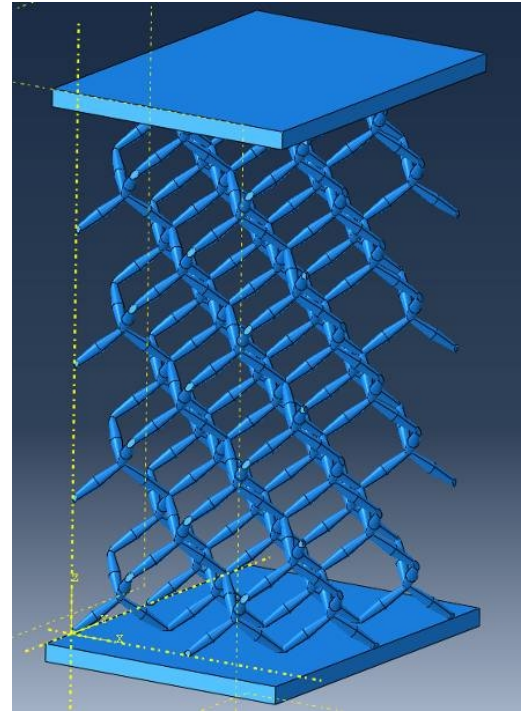

Fig.5 Parametrized modelling of a pentamode metamaterial model $(2 \times 2 \times 4)$

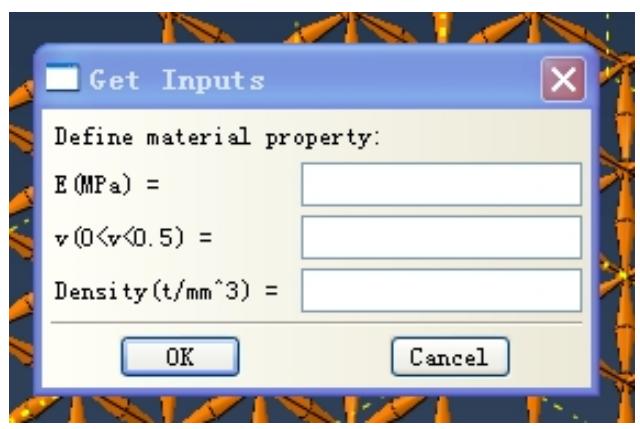

Fig. 6 Interactive input of material parameters

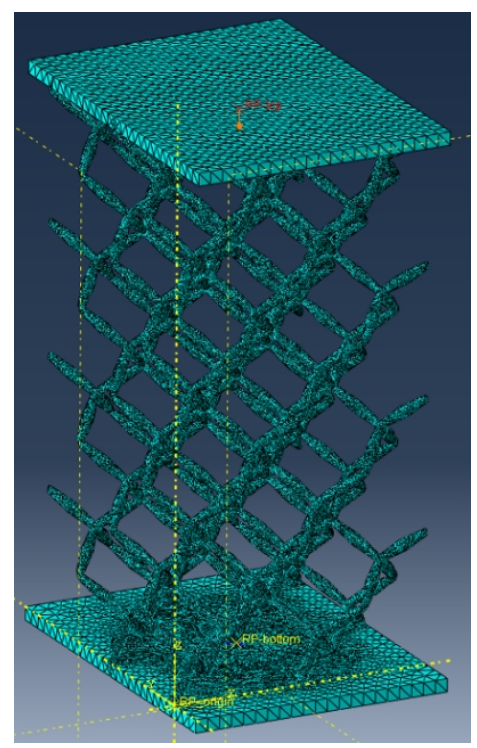

Fig.7 The FEA model of 3D pentamode metamaterials $(2 \times 2 \times 4)$

\section{Automatic Pre-processing}

Besides directly keying in parameters on the interactive input interfaces for parameterized modelling and material properties defining, other pre-processes, such as specifying material properties, grid partition, creation of coupling constraints, analysis step creation and output, creation 
of boundary conditions, and job creation and submission, are automatically completed by the Python programming language. Fig.7 shows the FEA model of 3D pentamode metamaterials.

\section{Rapid Post-processing Analysis of Metamaterials}

Based on the post-processing module of Python language, the post-processing of simulation results can be simplified so as to shorten the post-processing time. In this paper, the mechanical properties simulation aims to obtain the Young's modulus $E$ and the shear modulus $G$ corresponding to the $3 \mathrm{D}$ pentamode metamaterials, and the theoretical formula is used to calculate the bulk modulus $B$. Therefore, the post-processing module is mainly used for extracting the calculated model deformation and acting force and calculating the stress-strain curve by the stress and strain formulae, thus obtaining the Young's modulus $E$ and the shear modulus $G$.

According to the rapid post-processing analysis module, 10 groups of pentamode metamaterial mechanical properties simulation results under different parameters are obtained as shown in Tab.1.

Tab.1 Simulation results of mechanical properties of 3D pentamode metamaterials

\begin{tabular}{cccccccccccc}
\hline \multirow{2}{*}{ Group } & $p$ & $a$ & $D$ & $d$ & $D / a$ & $d / a$ & $E$ & $G$ & $B$ & $E / G$ & $B / G$ \\
\cline { 2 - 12 } & - & \multicolumn{9}{c}{$(\mathrm{mm})$} & \multicolumn{7}{c}{$(\%)$} & & $\mathrm{MPa}$ & & - & - \\
\hline 1 & 0.25 & 15 & 1.32 & 0.25 & 8.8 & 1.7 & 10 & 2.2 & 33.7 & 4.55 & 15.3 \\
2 & 0.25 & 15 & 1.32 & 0.5 & 8.8 & 3.3 & 68.3 & 15.1 & 57.9 & 4.52 & 3.8 \\
3 & 0.25 & 15 & 1.32 & 0.71 & 8.8 & 4.7 & 197.6 & 43.3 & 82.3 & 4.56 & 1.9 \\
4 & 0.25 & 15 & 1.32 & 1 & 8.8 & 6.7 & 507.3 & 105.9 & 101.5 & 4.79 & 1.0 \\
5 & 0.25 & 15 & 1.32 & 1.32 & 8.8 & 8.8 & 1025.8 & 211.1 & 116.1 & 4.86 & 0.6 \\
6 & 0.25 & 15 & 1.5 & 1.5 & 10 & 10 & 1805.2 & 366.1 & 146.3 & 4.93 & 0.4 \\
7 & 0.25 & 15 & 1 & 1 & 6.7 & 6.7 & 339.4 & 70.3 & 77.4 & 4.83 & 1.1 \\
8 & 0.25 & 15 & 1.8 & 1 & 12 & 6.7 & 788.6 & 168.2 & 138.0 & 4.69 & 0.8 \\
9 & 0.25 & 15 & 0.71 & 0.71 & 4.7 & 4.7 & 94.6 & 19.5 & 50.5 & 4.85 & 2.6 \\
10 & 0.25 & 15 & 0.3 & 0.2 & 2 & 1.3 & 0.92 & 0.2 & 10.1 & 4.6 & 50.3 \\
\hline
\end{tabular}

\section{Summary}

(1) A rapid modeling analysis approach of 3D pentamode metamaterials is proposed on the basis of Abaqus software using the Python script language. This paper makes an analysis of the pentamode metamaterial model parameters, constructs the programming procedure, embeds the inner loop and adds interactive input interfaces, thus not only ensuring simplicity and easy realization of program structure, but also satisfying the requirements of parameter input and loop operations.

(2) Besides interactive input interfaces for inputting parameters for the model and materials, other pre-processes and post-processes are automatically completed by the modules. This both makes sure that the parameterized modelling of metamaterials is simple and clear and avoids fully manual operations for the FEA modelling analysis, thus saving a lot of time and effort.

(3) The rapid modelling analysis approach can be transplantable and provide reference for simple 2D planar model and complex 3D entity model. Because the approach can realize the parameter-driven modelling, in the future it lays a foundation for further optimized design of the 3D pentamode metamaterial microstructure.

\section{Acknowledgement}

This research is financially supported by National Natural Science Fundation of China (No. 11502176) and Hubei Natural Science Fundation of China (No. 2014CFB23F). 


\section{References}

[1] Layman C N, Naify C J, Martin $\quad T \quad P$ et al.. Highly-anisotropic elements for acoustic pentamode applications [J]. http://arxiv.org/abs/1211.6698v1, 2012, 1-7.

[2] Qing Y J. Research of the microwave absorption properties of microwave absorbing material enhanced and tuned by metamaterials [D]. Changsha: Hunan Univercity, 2010. (in Chinese)

[3] Xiao Q J. Research on layered design of ring-shaped acoustic cloaking using bimode Metamaterial [J]. Applied Mechanics and Materials, 2014, 687-691: 4399-4404.

[4] Xiao Q J. Study on Design Method of the Microstructure for Layered and Ring-shaped Acoustic Cloaking Based on Bimode Metamaterial [J]. Applied Mechanics and Materials, 2014, 684: 219-227.

[5] Chen H Y. Acoustic cloaking in three dimensions using acostic metamaterials [J]. Applied Physics Letters, 2007, 91: 183518/1-183518/3.

[6] Shen H J, Wen J H, Yu D L, et al.. Research on a cylindrical cloak with active acostic metamaterial layers[J]. Acta Phys. Sin., 2012, 61(13): 134303/1-134301/8. (in Chinese)

[7] Cummer S A and Schuring D. One path to acoustic cloaking [J]. New Journal of Physics, 2007, 9(45): $1-8$.

[8] Wu L H. Effective nonlinear electromagnetic properties of 3-D metamaterials [J]. Journal of Harbin Institue of Technology, 2010, 42(9): 1509-1512.

[9] Zhou J. Applications of metamaterials in electronic components [J]. Electronic Components and Materials, 2008, 27(9): 1-4.

[10] Schittny R. Elastic measurements on macroscopic three-dimensional pentamode metamaterials [J]. Applied Physics Letters, 2013, 103: 231905/1-231905/4.

[11] Kadic M. On the practicability of pentamode mechanical metamaterials [J]. Applied Physics Letters, 2012, 100: 191901/1-191901/4.

[12] Cao J F. Applications of Python langerage in software Abaqus [M]. Beijing: China Machine Press, 2011. 